\title{
Odorant Receptor from the Southern House Mosquito Narrowly Tuned to the Oviposition Attractant Skatole
}

\author{
David T. Hughes • Julien Pelletier • Charles W. Luetje • \\ Walter S. Leal
}

Received: 3 June 2010 /Revised: 22 June 2010 / Accepted: 25 June 2010 / Published online: 11 July 2010

(C) The Author(s) 2010. This article is published with open access at Springerlink.com

\begin{abstract}
Oviposition attractants are environmental cues that allow Culex gravid female mosquitoes to locate suitable sites for egg-laying and, therefore, may be exploited for environmentally friendly strategies for controlling mosquito populations. Naturally occurring skatole has been identified as an oviposition attractant for the Southern House mosquito, Culex quinquefasciatus. Previously, we identified in $C x$. quinquefasciatus female antennae an olfactory receptor neuron (ORN) highly sensitive to skatole and an odorant-binding protein involved in the detection of this semiochemical. Here, we describe the characterization of an odorant receptor (OR), CquiOR10, which is narrowly tuned to skatole when expressed in the Xenopus oocyte system. Odorant-induced response profiles generated by heterologously expressed CquiOR10 suggest that this OR is expressed in the mosquito ORN sensitive to skatole. However, geranylacetone, which stimulates the antennal ORN, was not detected by CquiOR10-expressing oocytes, thus raising interesting questions about reception of oviposition attractants in mosquitoes.
\end{abstract}

Key Words Odorant receptor CquiOR10 · Culex quinquefasciatus Xenopus oocyte expression system . 3-Methylindole $\cdot 2$-Methylphenol

D. T. Hughes $\cdot$ C. W. Luetje

Department of Molecular and Cellular Pharmacology, University of Miami Miller School of Medicine,

Miami, FL, USA

\section{J. Pelletier $\cdot$ W. S. Leal $(\square)$}

Department of Entomology, University of California Davis,

1 Shields Ave,

Davis, CA 95616, USA

e-mail: wsleal@ucdavis.edu

\section{Introduction}

Culex mosquitoes are vectors of pathogens including the human filarial nematode, Wuchereria bancrofti, and encephalitis-causing viruses, such as St. Louis, Japanese, Venezuela equine, Western equine encephalitis, and West Nile virus (Nasci and Miller, 1996). ${ }^{1}$ Given the resistance of Culex populations to modern insecticides, alternative methods of controls are sorely needed. Larval development is a particularly vulnerable phase in the life cycle of Culex mosquitoes, as eggs are laid in rafts from which hundreds of larvae emerge in confined areas-thus facilitating management. Gravid females rely on environmental oviposition attractants to locate oviposition sites. Skatole, a natural product found in animal excreta and also a product of fermentation of organic material, has been identified as an oviposition attractant for the Southern House mosquito, Culex quinquefasciatus (Millar et al., 1992). Field studies have demonstrated that traps baited with optimal doses of skatole collected significantly more eggs (Mboera et al., 2000) and gravid females (Leal et al., 2008) than control traps, thus suggesting that in combination with a biological agent, Bacillus thuringiensis var. israelensis (Barbosa et al., 2010) oviposition attractants may be used in "attract-and-kill" strategies. Chemical ecology and olfaction are the pillars of these semiochemical-based, environmentally friendly strategies. Therefore, identification of olfactory proteins involved in the reception of these semiochemicals may open the door for development of better oviposition attractants. Recently, we demonstrated by RNA interference that an odorant-binding protein (OBP) from $C x$. quinquefasciatus, CquiOBP1, is involved directly in the

\footnotetext{
$\overline{{ }^{1} \text { We apologize }}$ for not being able to cite all the relevant literature due to reference limitations of rapid communication format.
} 
reception of skatole and other oviposition attractants (Pelletier et al., 2010a). We also have characterized an odorant receptor (OR) from this mosquito species, CquiOR2, which is highly sensitive to indole and moderately sensitive to skatole (Pelletier et al., 2010b). Here, we characterize CquiOR10 and show this OR to be highly sensitive and narrowly tuned to skatole.

\section{Methods and Materials}

Expression of CquiOR10 in the Xenopus Oocyte System Oocytes were prepared as previously described (Pelletier et al., 2010b). CquiOR10 and CquiOR7, initially cloned into pBlueScript (Pelletier et al., 2010b), were transferred to pGEMHE for use as templates for synthesis of capped cRNA by using mMessage mMachine kits (Ambion). Twenty-five ng of cRNA encoding each OR subunit were injected into Stage V-VI Xenopus oocytes. Oocytes were incubated at $18^{\circ} \mathrm{C}$ in Barth's saline (in mM: $88 \mathrm{NaCl}, 1$ $\mathrm{KCl}$, 2.4 NaHCO3, 0.3 CaNO3, 0.41 CaCl2, 0.82 MgSO4, 15 HEPES, $\mathrm{pH} 7.6$, and $100 \mu \mathrm{g} / \mathrm{ml}$ amikacin) for $2-5 \mathrm{~d}$ prior to electrophysiological recording.

Electrophysiology and Data Analysis Odorant-induced currents were recorded under two-electrode voltage clamp from oocytes expressing ORs, by using an automated parallel electrophysiology system (OpusXpress 6000A; Molecular Devices). Oocytes were perfused with ND96 (in mM: $96 \mathrm{NaCl}, 2 \mathrm{KCl}, 1 \mathrm{CaCl} 2,1 \mathrm{MgCl} 2,5$ HEPES, $\mathrm{pH}$ 7.5). Odorants were diluted in ND96 and applied for $20 \mathrm{sec}$ at a flow rate of $1.65 \mathrm{ml} / \mathrm{min}$ with extensive washing in ND96 (5-20 $\mathrm{min}$ at $4.6 \mathrm{ml} / \mathrm{min}$ ) between applications. Current responses approached a plateau during the $20 \mathrm{sec}$ application (Pelletier et al., 2010b). Micropipettes were filled with $3 \mathrm{M}$ $\mathrm{KCl}$ and had resistances of 0.2-2.0 $\mathrm{M} \Omega$. The holding potential was $-70 \mathrm{mV}$. Current responses were filtered (4-pole, Bessel, low pass) at $20 \mathrm{~Hz}(-3 \mathrm{db})$, sampled at $100 \mathrm{~Hz}$, and were captured and stored with OpusXpress 1.1 software (Molecular Devices). Initial analysis of electrophysiological data was done with Clampfit 9.1 software (Molecular Devices). Curve fitting of concentrationresponse data was done with Prism 4 (Graphpad).

\section{Results and Discussion}

In our search for molecular targets that may be used in a reverse chemical ecology approach for the development of better oviposition attractants (Leal et al., 2008), we recently have mined the genome of $C x$. quinquefasciatus and identified an OR sensitive to indole, CquiOR2, which also responded to methylindoles, including skatole (IUPAC name, 3-methylindole). By mapping the antennae of female $C x$. quinquefasciatus, we previously observed that a skatoledetecting ORN also responded to geranylacetone and ethyl hexanoate, but not indole (Syed and Leal, 2009). These observations prompted us to examine the odorant response profile of CquiOR10, an OR closely related to CquiOR2 (Pelletier et al., 2010b). Full-length coding sequence of CquiOR10 and the obligatory co-receptor CquiOR7 (Pelletier et al., 2010b) were cloned in pGEMHE for heterologous expression in Xenopus oocytes.

To identify the best ligand for this receptor, oocytes expressing CquiOR10 + CquiOR7 were screened first with a panel of odorants (Fig. 1a), each applied for $20 \mathrm{sec}$ at a concentration of $10 \mu \mathrm{M}$ with extensive washing between applications. Skatole (3-methylindole) elicited the largest current responses, but the receptor also responded with lower sensitivity to indole, other methylindoles, and 2-methylphenol. Interestingly, CquiOR10 was unresponsive to many compounds in the test panel of 23 odorants, including other oviposition attractants such as trimethylamine, nonanal, and the mosquito oviposition pheromone (MOP) (Leal et al., 2008).

Next, we performed concentration-response analyses for skatole and two other ligands, indole and 2-methylphenol, which were identified as the best ligands among the indoles and phenols, respectively, for the related receptor CquiOR2 (Pelletier et al., 2010b). Skatole was the most potent of these compounds, activating the CquiOR10 + CquiOR7 receptor with an $\mathrm{EC}_{50}$ of $90 \mathrm{nM}$. Indole and 2-methylphenol were less potent, activating CquiOR10 + CquiOR7 with $\mathrm{EC}_{50}$ values of $2.4 \mu \mathrm{M}$ and $41 \mu \mathrm{M}$, respectively. Interestingly, indole and 2-methylphenol also displayed lower efficacy (maximal response) than skatole ( $40 \pm 2 \%$ and $69 \pm 3 \%$ of skatole, respectively). In addition, the response threshold for skatole was two to three orders of magnitude higher than that observed for indole and 2-methylphenol (Fig. 1b, c). Thus, we found that heterologously expressed CquiOR10 is highly sensitive and narrowly tuned to the oviposition attractant skatole.

In female antennae of the Southern House mosquito, skatole is detected by a small-spike-amplitude ORN housed in A1 sensilla (Syed and Leal, 2009), which is also sensitive to a lower degree to geranylacetone and ethyl hexanoate, but does not respond to indole or 2methylphenol [see Figs. S5, S6, supporting information in (Syed and Leal, 2009)]. In the Xenopus oocyte system, CquiOR10 was unresponsive to geranylacetone. Although expression of ORs in heterologous systems, such as the Xenopus oocyte system, is an invaluable tool for deorphanizing and characterizing receptors, it does not completely mimic the insect olfactory system. Typically, these systems are devoid of OBPs, odorant-degrading enzymes, sensory neuron membrane proteins, and other 


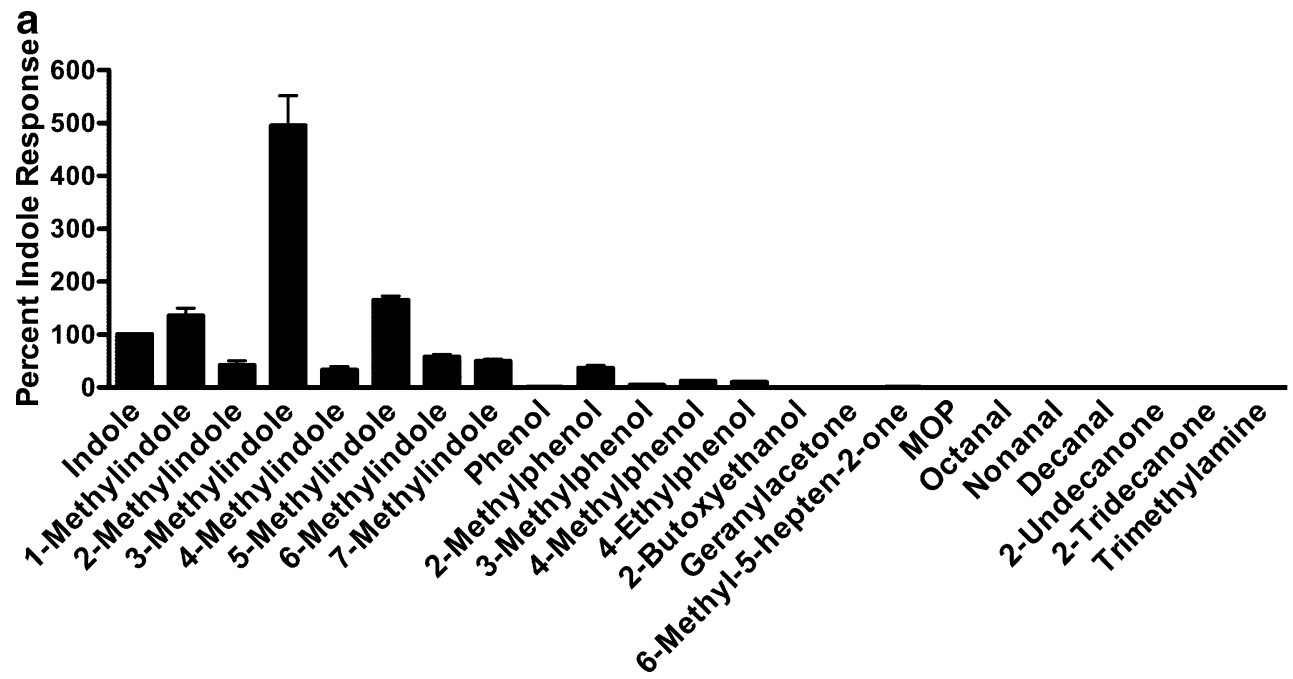

b

3-Methylindole (nM)

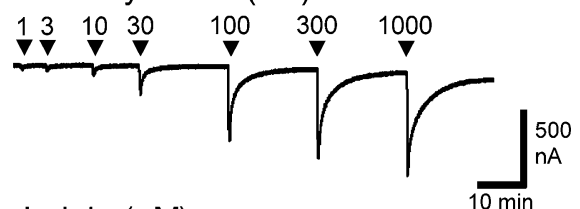

Indole $(\mu \mathrm{M})$

2-Methylphenol $(\mu \mathrm{M})$

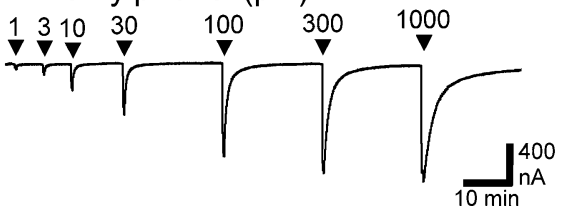

Fig. 1 Odorant receptor CquiOR10 is highly sensitive to skatole (3-methylindole). a Oocytes expressing CquiOR10 + CquiOR7 were challenged with a panel of odorant compounds. Each odorant was applied at a concentration of $10 \mu \mathrm{M}$ for $20 \mathrm{sec}$ with $10 \mathrm{~min}$ washes between applications. All responses are normalized to the response of the same oocyte to $10 \mu \mathrm{M}$ indole (mean \pm SEM, $N=4-5$ ). b Oocytes expressing CquiOR10 + CquiOR7 were challenged with a range of concentrations of 3-methylindole (top trace), indole (middle trace) or 2-methylphenol (bottom trace). Each odorant was applied for $20 \mathrm{sec}$ with 5-20 min washes between applications. Note different scales: from top to bottom 500, 200 and $400 \mathrm{nA}$. c Oocytes expressing

olfactory proteins that may play a part in the selectivity and sensitivity of the olfactory system. Thus, it is conceivable that heterologously expressed CquiOR10 and the receptor in its native environment differ in the detection of geranylacetone because the former is devoid of OBPs. However, one cannot rule out the possibility that a separate ORN responding to geranylacetone has the same spike amplitude as the skatole-detecting ORN (Syed and Leal,
C
- 3-methylindole
- Indole
$\square$ 2-methylphenol

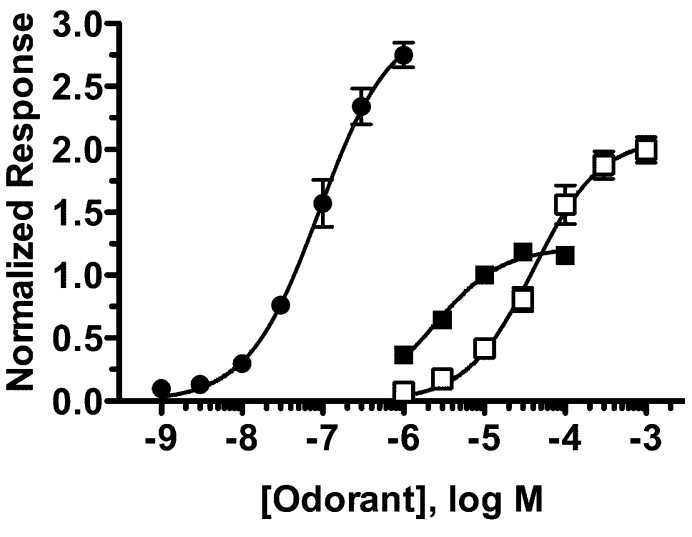

CquiOR10 + CquiOR7 were challenged with a range of concentrations of 3-methylindole, indole, and 2-methylphenol. Responses were normalized to the response of each oocyte to $10 \mu \mathrm{M}$ indole and are presented as mean \pm sem $(N=3-5$ for each odorant tested). Data were fit to the equation: $\mathrm{I}=\mathrm{I}_{\max } /\left(1+\left(\mathrm{EC}_{50} / \mathrm{X}\right)^{\mathrm{n}}\right)$ where I represents the current response at a given concentration of odorant (X), $\mathrm{I}_{\max }$ is the maximal response, $\mathrm{EC}_{50}$ is the concentration of odorant yielding a half maximal response, and $\mathrm{n}$ is the apparent Hill coefficient. Derived values are: 3 -methylindole, $\mathrm{EC}_{50}=90 \pm 17 \mathrm{nM}, N=1.0 \pm 0.1$; indole, $\mathrm{EC}_{50}=2.4 \pm 0.3 \mu \mathrm{M}, N=1.1 \pm 0.2 ; 2$-methylphenol, $\mathrm{EC}_{50}=41 \pm 7 \mu \mathrm{M}$, $N=1.0 \pm 0.1$

2009), thus rendering them indistinguishable by single unit recordings. Alternatively, the same small-spike neuron sensitive to skatole may express another OR along with CquiOR10. In marked contrast to the mammalian olfactory system, co-expression of ORs has been documented in Drosophila melanogaster. Co-expression of CquiOR10 and another OR would not be entirely surprising given the number of putative odorant receptors in the Southern House 
mosquito genome (Pelletier et al., 2010b) and the number of ORNs in their sensory system (Syed and Leal, 2007, 2008). Future research aimed at testing these three hypotheses might lead to deeper understanding of odorant reception in mosquitoes.

Acknowledgments This work was supported in part by the National Science Foundation (0918177 to W.S.L.), National Institutes of Health (DC011091 to C.W.L.) and gifts from Bedoukian Research Inc. and Fuji Flavor Co. (to W.S.L.). We thank lab members, particularly Drs. Zain Syed, Wei Xu, and Elizabeth Atungulu (UCD), for enlightening discussion, and Ana Castro and Vanessa Santos (UM) for help with oocyte preparation.

Open Access This article is distributed under the terms of the Creative Commons Attribution Noncommercial License which permits any noncommercial use, distribution, and reproduction in any medium, provided the original author(s) and source are credited.

\section{References}

Barbosa, R. M., Regis L., VAsconcelos R., and LeAL W. S. 2010. Culex mosquitoes (Diptera: Culicidae) egg laying in traps loaded with Bacillus thuringiensis variety israelensis and baited with skatole. J. Med. Entomol. 47:345-348.

Leal, W. S., Barbosa R. M., Xu W., Ishida Y., Syed Z., Latte N., Chen A. M., Morgan T. I., Cornel A. J., and Furtado A.
2008. Reverse and conventional chemical ecology approaches for the development of oviposition attractants for Culex mosquitoes. PLOS ONE 3:e3045.

Mboera, L. E., Takken W., Mdira K. Y., and Pickett J. A. 2000. Sampling gravid Culex quinquefasciatus (Diptera: Culicidae) in Tanzania with traps baited with synthetic oviposition pheromone and grass infusions. J. Med. Entomol. 37:172-176.

Millar, J. G., Chaney J. D., and Mulla M. S. 1992. Identification of oviposition attractants for Culex quinquefasciatus from fermented Bermuda grass infusions. J. Am. Mosq. Control Assoc. 8:11-17.

NASCI, R. S., and MiLler B. R. 1996. Culicine mosquitoes and the agents they transmit, pp 85-97, in B. J. Beaty and W. C. Marquardt (eds.). The Biology of Disease Vectors. University Press of Colorado, Niwot.

Pelletier, J., Guidolin A., Syed Z., Cornel A. J., and Leal W. S. 2010a. Knockdown of a mosquito odorant-binding protein involved in the sensitive detection of oviposition attractants. $J$. Chem. Ecol. 36:245-248.

Pelletier, J., Hughes D. T., Luetje C. W., and Leal W. S. 2010 b. An odorant receptor from the southern house mosquito Culex pipiens quinquefasciatus sensitive to oviposition attractants. PLOS ONE 5:e10090.

SYED, Z., and LEAL W. S. 2007. Maxillary palps are broad spectrum odorant detectors in Culex quinquefasciatus. Chem. Senses $32: 727-738$

Syed, Z., and LEAL W. S. 2008. Mosquitoes smell and avoid the insect repellent DEET. Proc. Natl. Acad. Sci. USA 105:1359813603.

Syed, Z., and LEAL W. S. 2009. Acute olfactory response of Culex mosquitoes to a human- and bird-derived attractant. Proc. Natl. Acad. Sci. USA 106:18803-18808. 